\title{
Hybrid wide-angle viewing-endoscopic vitrectomy using a 3D visualization system
}

This article was published in the following Dove Press journal:

Clinical Ophthalmology

\section{Mihori Kita \\ Yuki Mori \\ Sachiyo Hama}

Department of Ophthalmology, National Organization Kyoto Medical Center, Kyoto, Japan
Correspondence: Mihori Kita

Department of Ophthalmology, National Organization Kyoto Medical Center, I-I Mukouhata-cho, Fukakusa, Fushimi-ku, Kyoto 6|2-8555, Japan

$\mathrm{Tel}+81756419161$

Fax +8I 756434325

Email mihorik@kuhp.kyoto-u.ac.jp
Purpose: To introduce a hybrid wide-angle viewing-endoscopic vitrectomy, which we have reported, using a 3D visualization system developed recently.

Subjects and methods: We report a single center, retrospective, consecutive surgical case series of 113 eyes that underwent $25 \mathrm{G}$ vitrectomy (rhegmatogenous retinal detachment or proliferative vitreoretinopathy, 49 eyes; epiretinal membrane, 18 eyes; proliferative diabetic retinopathy, 17 eyes; vitreous opacity or vitreous hemorrhage, 11 eyes; macular hole, 11 eyes; vitreomacular traction syndrome, 4 eyes; and luxation of intraocular lens, 3 eyes).

Results: This system was successfully used to perform hybrid vitrectomy in the difficult cases, such as proliferative vitreoretinopathy and proliferative diabetic retinopathy.

Conclusion: Hybrid wide-angle viewing-endoscopic vitrectomy using a 3D visualization system appears to be a valuable and promising method for managing various types of vitreoretinal disease.

Keywords: $25 \mathrm{G}$ vitrectomy, endoscope, wide-angle viewing system, 3D visualization system, hybrid

\section{Introduction}

We have previously reported on the efficacy of combining the use of a wide-angle viewing system and an endoscope, called "hybrid vitrectomy".,

The recent development of a 3D heads-up visualization system has helped lead to further technological improvements in the ophthalmological field. ${ }^{3-6}$ This digital system not only enables access to 3D surgical images, but also makes it possible to freely manipulate the color and brightness of the images. Furthermore, multiple images, such as an endoscopic view and optical coherence tomography images, as well as examination data can be simultaneously integrated on the same monitor.

In this report, we introduce a hybrid wide-angle viewing-endoscopic vitrectomy procedure that uses a $3 \mathrm{D}$ visualization system.

\section{Subjects and methods}

This study was approved by the Ethics Committee of the National Hospital Organization Kyoto Medical Center, and all procedures involved adhered to the tenets of the Declaration of Helsinki. Written informed consent for the procedure and participating in our study was received from all the patients after explaining the surgical procedures.

We report a single center, retrospective, consecutive surgical case series of 113 eyes that underwent vitrectomy between May and October 2017. Indications for vitrectomy included rhegmatogenous retinal detachment or proliferative vitreoretinopathy (49 eyes), epiretinal membrane (18 eyes), proliferative diabetic retinopathy (17 eyes), vitreous 
opacity or vitreous hemorrhage (11 eyes), macular hole (11 eyes), vitreomacular traction syndrome (4 eyes), and luxation of intraocular lens (3 eyes). Phacoemulsification aspiration and intraocular lens implantation was simultaneously performed using a $3 \mathrm{D}$ visualization system in 90 eyes.

\section{Instruments and surgical procedures}

The Constellation Vision System including three valved trocars entrance (Alcon Laboratories, Fort Worth, TX, USA) was used to perform all of the $25 \mathrm{G}$ vitrectomy procedures. The NGENUITY 3D ${ }^{\circledR}$ visualization system (Alcon Laboratories) was attached to a VISU 210 microscope (Carl Zeiss Meditec, Jena, Germany) and used to observe all of the surgical procedures. A twin chandelier light system (Dutch Ophthalmic Research Center, Zuidland, the Netherlands) was used for the main intraocular illumination. The medical staff in the operating room wore circular polarized glasses to see in $3 \mathrm{D}$.

Images of the fundus on the 3D visualization system monitor were obtained through either a RESIGHT wideangle viewing system (Carl Zeiss Meditec) or an ocular endoscope system consisting of a high definition camera FC-304 (10K pixels in resolution), LED light unit FL-301 used as a supplemental intraocular illumination, $25 \mathrm{G}$ fiber Previt, and image processer FI-302 (Fiber Tec, Tokyo, Japan), or by using both systems (Figure 1). For example, in one of the retinal detachment cases, the core vitrectomy, creation of the posterior vitreous detachment, injection of the perfluorocarbon liquid, and the fluid/air exchange including the perfluorocarbon liquid removal were all visualized using a wide-angle viewing system. However, the peripheral

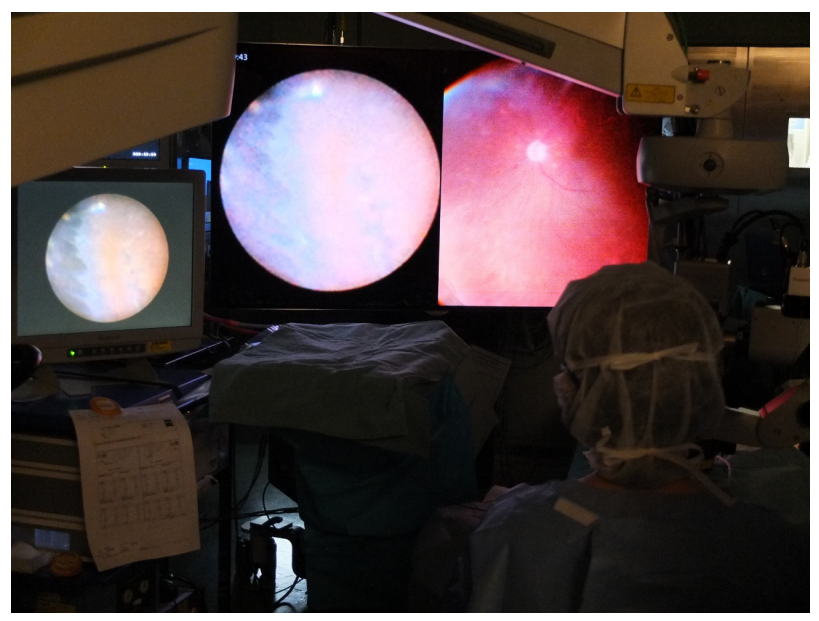

Figure I Both the endoscopic image (left) and the wide-angle viewing image (right) are integrated on the large 3D monitor that is placed at the foot of the bed. The small conventional monitor for the endoscope is placed on the left side of the surgeon. maneuvers, such as shaving the peripheral vitreous, identifying the retinal breaks, drainage of the subretinal fluid through the peripheral breaks, laser photocoagulation or cryopexy of the peripheral area, were all visualized using an endoscope (Figure 2, Video S1).

Membrane peeling at a posterior pole was visualized through the use of a magnifying contact lens that was placed on the cornea. In all of the cases, including the macular diseases, all 360 degrees of the peripheral fundus were inspected using an endoscope in order to detect any peripheral lesions or iatrogenic retinal breaks at the end of the intraocular procedure. A single surgeon (MK) performed all of the surgeries. All of the surgical staff, including the primary surgeon, wore polarized glasses during the surgical procedures.

\section{Results}

There were no cases that required conversion of the $3 \mathrm{D}$ visualization to a conventional microscopic view. In all surgeries, the $3 \mathrm{D}$ visualization system was successfully used to perform the hybrid vitrectomy, even in the difficult cases such as proliferative vitreoretinopathy and proliferative diabetic retinopathy. Illuminations of the microscope, chandelier light, and endoscope were reduced by $40 \%, 60 \%$, and $20 \%$ respectively.

Hole closure was achieved after the first operation in all of the macular hole cases. However, one of the proliferative vitreoretinopathy eyes had wide subretinal strands that subsequently required the patient to undergo a second vitrectomy in order to reattach the retina. Reattachment was achieved after the first vitrectomy in all of the other 48 eyes with retinal detachment or proliferative vitreoretinopathy. None of the patients exhibited any other intraoperative or postoperative complications.

\section{Discussion}

Although the wide-angle viewing system enables us to observe a panoramic fundus, the image is still small. In contrast, when using an endoscope, it is possible to enlarge the image by simply closing in on the retina, thereby facilitating the identification of any tiny preoperatively undetected retinal breaks. ${ }^{7}$ However, the observation field when using an endoscope is narrow, with the view normally being nonstereoscopic.

When using a wide-angle viewing system, indentation of the sclera is inevitable when trying to observe or manipulate the periphery, which can cause intraoperative pain and postoperative inflammatory reactions. In contrast, use of an endoscope makes it possible to observe the peripheral area 

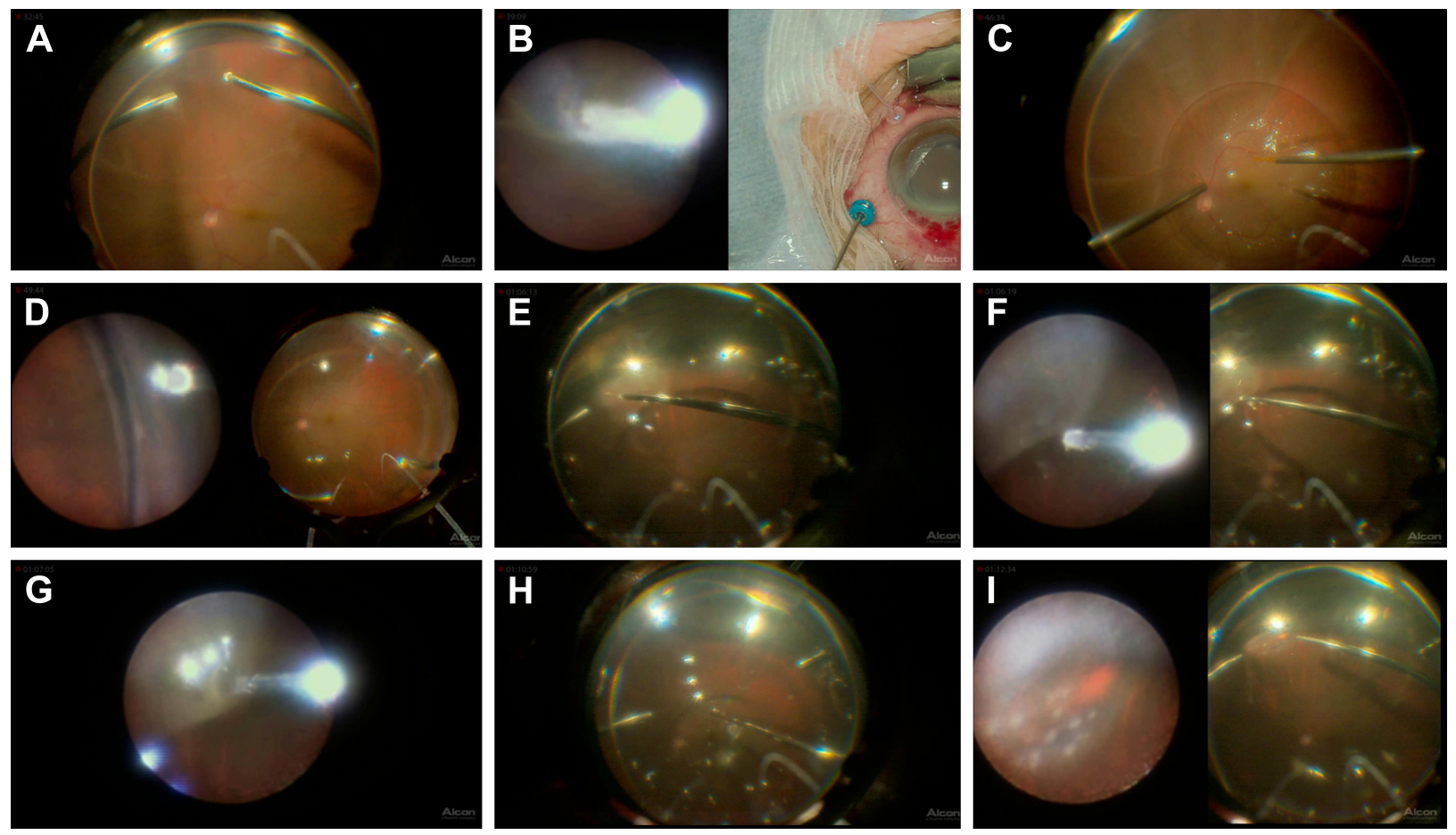

Figure 2 Intraoperative 2D snapshots in a case of hybrid surgery for rhegmatogenous retinal detachment. (A) Core vitrectomy performed using a wide-angle view (WAV). (B) Cryopexy to fix a tiny retinal break identified intraoperatively at the ora serrata using the endoscopic view (left). A microscopic view of the outer segment is simultaneously visualized (right) and helps to prevent freezing of the cornea or the lid. (C) Injection of perfluorocarbon liquid (PFCL) while using WAV. (D) Peripheral vitreous shaving performed while using the endoscopic view without any scleral depression. (E) Fluid/air exchange and removal of PFCL using WAV. (F) Internal drainage through an original retinal break at the periphery. The images from both the endoscopic view (left) and WAV (right) are integrated on the same monitor. (G) Endoscopic view of the internal drainage. (H) PFCL is completely removed while using WAV. (I) Laser photocoagulation of a retinal break while using the magnified endoscopic view (left). Simultaneous visualization by WAV (right) helps with the orientation of the endoscopic manipulation.

of the fundus and anterior part of the eye without any scleral indentation, ${ }^{8}$ which can contribute to a less invasive surgery. Therefore, to improve the efficiency of the procedure, the best visualization tool at each step of the surgery should be selected for each case.

Based on these issues, our previous studies have investigated and recommended the use of hybrid wide-angle viewing-endoscopic vitrectomy. ${ }^{1,2}$ Furthermore, a 360-degree inspection of the periphery using an endoscope at the end of the vitrectomy ensures that the surgery has been completed without any complications, which can help reduce the risk of reoperations.

The first reported use of digital heads-up surgical viewing in the neurological field occurred in $1997 .{ }^{9}$ In the ophthalmological field, however, the first reported use for cataract surgery was by Weinstock et al in $2008,{ }^{3}$ while Riemann reported using it for vitrectomy in 2010. ${ }^{4}$ The advantages of using this system compared to viewing the surgical field through a microscope include: 1) ergonomics of the surgeons due to the heads-up position; 2) educational capability; 3) reduction in the risk of phototoxicity; 4) real-time color manipulation; and 5) integration of several views on a large monitor.
When using the hybrid wide-angle viewing systemendoscopic vitrectomy, the surgeon is required to look back and forth between a microscope and a small monitor for the endoscopy. However, the digital 3D visualization system allows both views on one large monitor at the same time. This can help to make the surgeon more comfortable with regard to the ergonomics aspect, in addition to making the hybrid vitrectomy more efficient and safe.

As a 16:9 sized monitor is used for the digital 3D visualization system, it is difficult to observe the superior or inferior peripheral fundus different from that seen with the microscopic view. Thus, use of the endoscopic view can compensate for this and make it possible for smooth and efficient manipulations.

At the present time however, the endoscopic view on the monitor of this system is only 2D. To overcome this issue, a 3D convertor (Shinko Optical, Tokyo, Japan) is now commercially available, and makes it possible to change the $2 \mathrm{D}$ endoscopic images to 3D real-time images. In the near future $3 \mathrm{D}$ endoscopic images are expected to be integrated into the monitors of the system, which will allow for safer manipulation and more comfortable conditions for the surgeons. 


\section{Conclusion}

Hybrid wide-angle viewing-endoscopic vitrectomy using a $3 \mathrm{D}$ visualization system appears to be a valuable and promising method that can be used to manage various types of vitreoretinal disease, including complex cases.

\section{Disclosure}

The authors report no conflicts of interest in this work.

\section{References}

1. Morishita S, Kita M, Yoshitake S, Hirose M, Oh H. 23-gauge vitrectomy assisted by combined endoscopy and a wide-angle viewing system for retinal detachment with severe penetrating corneal injury: a case report. Clin Ophthalmol. 2011;5:1767-1770.

2. Kita M. Endoscope-assisted vitrectomy. World J Ophthalmol. 2014; 4(3):52-55.

3. Cataract and Refractive Surgery Today. [webpage on the Internet]. Weinstock RJ, Donnenfeld ED. 3D visualization in ophthalmology. 2008:62-65. Available from: https://crstoday.com/articles/2008-may/ crst0508_16-php/. Accessed January 24, 2018.
4. Riemann CD. Vision and vitrectomy three-dimensional high definition (3DHD) video for surgical visualization in the retina OR. Presented at: American Academy of Ophthalmology; October 16-19, 2010; Chicago, IL.

5. Eckardt C, Paulo EB. Heads-up surgery for vitreoretinal procedures: an experimental and clinical study. Retina. 2016;36(1):137-147.

6. Retina Today. [webpage on the Internet]. Yonekawa Y. Seeing the world through 3-D glasses. 2016;11:54-60. Available from: http://retinatoday. com/2016/10/seeing-the-world-through-3-d-glasses/. Accessed January 24, 2018.

7. Kita M, Yoshimura N. Endoscope-assisted vitrectomy in the management of pseudophakic and aphakic retinal detachments with undetected retinal breaks. Retina. 2011;31(7):1347-1351.

8. Yoshitake S, Oh H, Kita M. Endoscope-assisted vitrectomy for retinal detachment in an eye with microcornea. Jpn J Ophthalmol. 2012;56(6): 613-616.

9. Levy ML, Day JD, Albuquerque F, et al. Heads-up intraoperative endoscopic imaging: a prospective evaluation of techniques and limitations. Neurosurgery. 1997;40:526-530. 


\section{Supplementary material}

Video S1 shows intraoperative side-by-side stereo image in a case of hybrid surgery for rhegmatogenous retinal detachment (same case as shown in Figure 2). Core vitrectomy was performed while using a wide-angle view (WAV). Cryopexy was applied to a tiny retinal break identified intraoperatively at the ora serrata when using the endoscopic view (left). Simultaneous visualization of the microscopic view for the outer segment (right) helps to prevent freezing the cornea or the lid. Perfluorocarbon liquid (PFCL) was injected while using WAV. Peripheral vitreous shaving was performed using the endoscopic view without any scleral depression. Fluid/air exchange and removal of PFCL was performed while using WAV, followed by internal drainage through an original retinal break at the periphery. Images from both the endoscopic view (left) and WAV (right) are integrated on the same monitor. Also, the endoscopic view alone can be monitored. PFCL is completely removed while using WAV. Laser photocoagulation of a retinal break while using the magnified endoscopic view (left). Simultaneous visualization of the WAV helps with orientation of the endoscopic manipulation.

\section{Publish your work in this journal}

Clinical Ophthalmology is an international, peer-reviewed journal covering all subspecialties within ophthalmology. Key topics include: Optometry; Visual science; Pharmacology and drug therapy in eye diseases; Basic Sciences; Primary and Secondary eye care; Patien Safety and Quality of Care Improvements. This journal is indexed on

Submit your manuscript here: http://www.dovepress.com/clinical-ophthalmology-journal
PubMed Central and CAS, and is the official journal of The Society of Clinical Ophthalmology (SCO). The manuscript management system is completely online and includes a very quick and fair peer-review system, which is all easy to use. Visit http://www.dovepress.com/ testimonials.php to read real quotes from published authors. 